\title{
Rabdomioma cardiaco en relación con tetralogía de Fallot y esclerosis tuberosa
}

\section{Cardiac rhabdomyoma associated with tetralogy of Fallot and tuberous sclerosis}

\author{
Olga C. Maza-Caneva', Óscar Osorio-Carbono², Margarita Bolaño-Esquiroß ${ }^{3}$, \\ Anabelly Delgado-Montenegro 4 y Sammy O. Ariza-Mendoza ${ }^{4 *}$
}

${ }^{1}$ Cardiología Pediátrica, Organización Clínica General del Norte, Barranquilla; 2 Servicio de Neonatología, Fundación Hospital Universitario Metropolitano, Barranquilla; ${ }^{3}$ Departamento de Pediatría, Universidad del Sinú-Seccional Cartagena, Cartagena; ${ }^{4}$ Departamento de Pediatría, Universidad Metropolitana, Barranquilla. Colombia

\section{Resumen}

Los tumores cardiacos son inhabituales en la edad pediátrica; de ellos, el rabdomioma es el más prevalente. Su curso suele ser benigno. Los casos sintomáticos obedecen a su efecto mecánico obstructivo o a la presencia de arritmias. No es común su asociación con cardiopatías congénitas. Se presenta el caso de un lactante con rabdomiomas múltiples dentro del complejo de esclerosis tuberosa y portador de tetralogía de Fallot con estenosis infundibulovalvular grave, quien presentó crisis de hipoxia que requirió valvuloplastia pulmonar percutánea, la cual se complicó por arritmia supraventricular y tuvo un desenlace fatal.

Palabras clave: Tumores cardiacos primarios. Tetralogía de Fallot. Rabdomioma. Esclerosis tuberosa.

\begin{abstract}
Cardiac tumors are rare in pediatrics, and when they occur, rhabdomyomas are the most prevalent. They are generally benign; symptomatic cases are due to mechanical obstruction or arrhythmias. They are not commonly associated with congenital heart disease. We present the case of an infant with multiple rhabdomyomas as part of tuberous sclerosis complex, and tetralogy of Fallot with severe infundibular and valvular stenosis, who presented a hypoxic crisis requiring percutaneous pulmonary valvuloplasty which was complicated by supraventricular arrythmia and had a fatal outcome.
\end{abstract}

Key words: Primary cardiac tumors. Tetralogy of Fallot. Rhabdomyoma. Tuberous sclerosis.

\section{Introducción}

Los tumores cardiacos primarios son raros en la población pediátrica. Tienen una tasa de incidencia que varía entre el $0.027 \%$ y el $0.08 \%$. El más prevalente es el rabdomioma, el cual tiene una mayor incidencia durante el primer año de vida y una baja tasa de mortalidad. Su curso suele ser asintomático y tiene resolución espontánea en el $90 \%$ de los casos ${ }^{1}$.

Los rabdomiomas cardiacos son tumores benignos que se originan de las fibras miocárdicas. Se han descrito varias formas de presentación clínica; la más importante dentro del complejo es la esclerosis tuberosa,

\section{Correspondencia:}

*Sammy O. Ariza-Mendoza

E-mail: orangel_36@hotmail.com
Fecha de recepción: 12-10-2019

Fecha de aceptación: 26-05-2020

DOI: 10.24875/RCCAR.M21000089
Disponible en internet: 29-11-2021 Rev Colomb Cardiol. 2021;28(5):489-494 www.rccardiologia.com 0120-5633 / @ 2020 Sociedad Colombiana de Cardiología y Cirugía Cardiovascular. Publicado por Permanyer. Este es un artículo open access bajo la licencia CC BY-NC-ND (http://creativecommons.org/licenses/by-nc-nd/4.0/). 
definida como una afección multisistémica de origen genético hereditario (autosómico dominante) de alta expresividad y penetrancia, pero con una baja incidencia, que oscila entre $1 / 5000$ y 1/10,000 nacidos vivos. Clínicamente se caracteriza por el desarrollo de trastornos neurológicos (crisis convulsivas), lesiones cutáneas (máculas hipocrómicas, angiofibromas faciales, fibromas ungueales) y orales (hipoplasia del esmalte dental), y hamartomas que pueden afectar el sistema nervioso central (túberes corticales o subependimarios), la retina, la cavidad oral, el corazón (rabdomioma), el pulmón y el riñón ${ }^{2}$. En ocasiones, los rabdomiomas cardiacos pueden presentarse como una lesión esporádica aislada y estar asociados a otras enfermedades o malformaciones cardiacas congénitas ${ }^{3}$. La localización más común es el ventrículo izquierdo, principalmente en la zona septal o apical, pero pueden darse en cualquier otra región del corazón. En la mayoría de los casos son múltiples y de tamaño variable 4 .

El rabdomioma asociado a otras malformaciones cardiacas es infrecuente. Su expresión sintomática es el resultado de la suma de los efectos combinados de las alteraciones estructurales congénitas y los efectos obstructivos o arritmogénicos de la masa intracardiaca.

La tetralogía de Fallot se considera la cardiopatía cianógena más frecuente, y ocurre en 1 de cada 3500 nacidos vivos. Es una malformación de origen troncoconal, definida por cuatro componentes: estenosis pulmonar, comunicación interventricular, cabalgamiento de la aorta e hipertrofia ventricular derecha. Su origen es multifactorial y puede asociarse en un $15-25 \%$ de los casos con anormalidades cromosómicas, en mayor relación con la trisomía 21 y la deleción 22Q11. La expresión clínica de la tetralogía de Fallot dependerá de la gravedad de la estenosis pulmonar y fluctuará desde la forma extrema, que cursa con cianosis desde el nacimiento y crisis hipóxicas en mayores de 1 mes, hasta la rosada 5 . Habitualmente, los pacientes tienen síntomas durante el tercer mes de vida. La reparación quirúrgica se realiza en el $90 \%$ de los casos entre los 30 días y el primer año de edad, la mayoría entre los 3 y 6 meses. En recién nacidos y lactantes con estenosis grave se plantea la valvuloplastia pulmonar como procedimiento paliativo ${ }^{6}$.

Como se mencionó, la relación del rabdomioma con la tetralogía de Fallot es infrecuente y son pocos los casos reportados en la literatura. Su abordaje dependerá de la expresión clínica de cada una de las enfermedades, planteándose en primera instancia una reparación quirúrgica de las alteraciones estructurales de la tetralogía de Fallot y la resección quirúrgica de los rabdomiomas, siempre que estos generen efecto

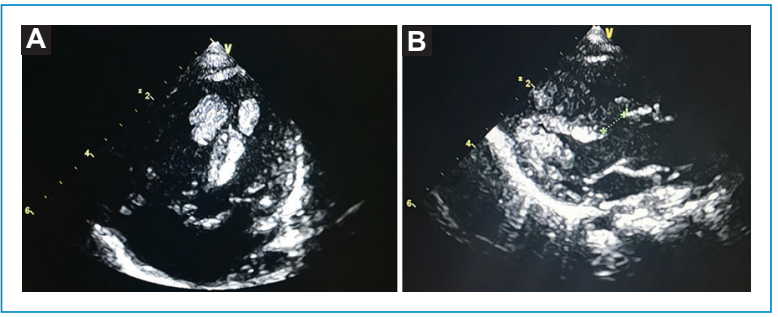

Figura 1. Ecocardiograma Doppler color. Tetralogía de Fallot con cuatro masas intracardiacas biventriculares. A: se observan imágenes hiperecogénicas en numero de 3 , bien definidas y diferenciadas a lado y lado de septum interventricular, compatibles con rabdomiomas. B: se observan las cavidades izquierdas en un eje largo con presencia de imagen hiperecogénica que compromete la porción media y apical del VI.

obstructivo o arritmogénico que comprometa el gasto cardiaco y, por tanto, la sobrevida del paciente?

\section{Caso clínico}

Varón de 1 mes de edad que ingresa referido a nuestro centro con diagnóstico de dos masas intracardiacas, localizadas en la porción apical del ventrículo derecho $(5 \times 7 \mathrm{~mm})$ y la pared septal del ventrículo izquierdo $(9 \times 4 \mathrm{~mm})$, sin descripción de repercusión hemodinámica ni cardiopatía estructural.

A su ingreso, un ecocardiograma transtorácico reporta comunicación interventricular subaórtica de $6 \mathrm{~mm}$ de diámetro, con cortocircuito bidireccional, aorta cabalgando un $50 \%$ del tabique interventricular, válvula pulmonar displásica $(Z$-1.1) con velocidad máxima de $3.5 \mathrm{~m} / \mathrm{s}$ y gradiente máximo de $50 \mathrm{mmHg}$, tronco y ramas pulmonares pequeñas $(Z-2)$ alcanzando una velocidad máxima de $4.7 \mathrm{~m} / \mathrm{s}$ y gradiente máximo de $90 \mathrm{mmHg}$, por lo que se concluye tetralogía de Fallot con estenosis pulmonar valvular y supravalvular graves. Además, se confirman las masas cardiacas intramurales bien circunscritas, mayoritariamente en el ventrículo izquierdo, donde se describen tres adosadas al tabique interventricular, en el tracto de entrada de $12.4 \times 7.7 \mathrm{~mm}$, en la porción media extendida al ápex de $14 \times 4.5 \mathrm{~mm}$ y apical de $10 \times 5 \mathrm{~mm}$, y en el ventrículo derecho una apical de $11.5 \times 5.3 \mathrm{~mm}$ de diámetro sin generar obstrucción mecánica, impresionando rabdomiosarcomas (Fig. 1).

Se realiza resonancia magnética (RM) cerebral como búsqueda activa del complejo de esclerosis tuberosa y se constata la presencia de múltiples y pequeños túberes subependimarios en los márgenes del sistema ventricular lateral (Fig. 2). Se realizó ecografía 


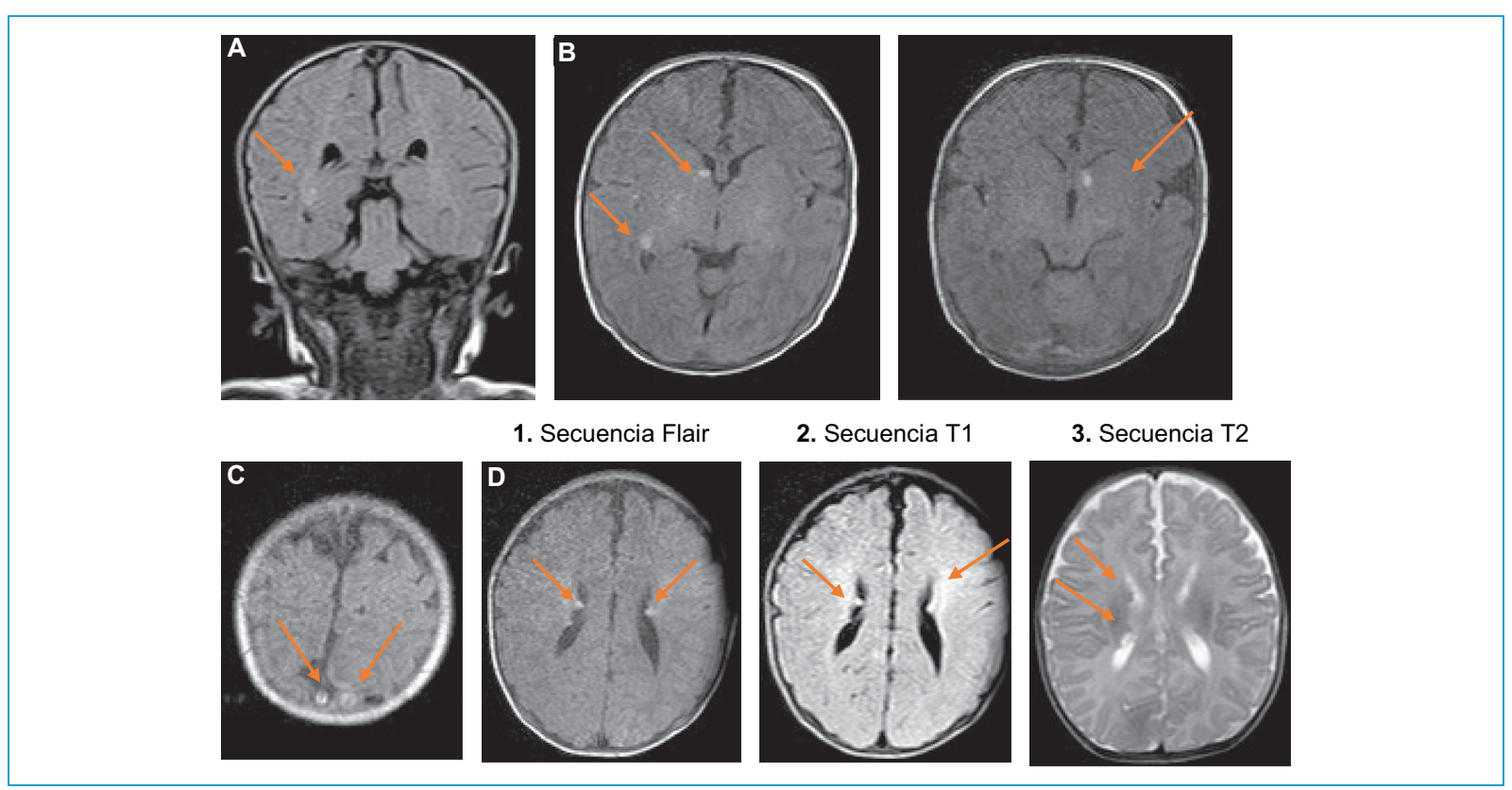

Figura 2. Resonancia magnética cerebral. A: corte coronal que muestra un nódulo periventricular derecho. B: corte transversal que muestra múltiples nódulos gangliobasales bilaterales. C: corte transversal que muestra nódulos parietooccipitales en la línea media. D: nódulos subependimarios en los márgenes del sistema ventricular lateral.

abdominal total que no mostró lesiones ni masas en otros órganos, complementándose con ecografía renal y de vías urinarias, en la que se evidenciaron unos riñones de tamaño, contornos y ecogenicidad normales, y se descartaron masas sólidas o quísticas.

Como antecedente de importancia, la madre tiene historia de crisis convulsivas en la infancia temprana y en su inspección se hallaron angiofibromas en el rostro y el tronco, máculas hipopigmentadas en hojas de fresno en la región dorsolumbar izquierda e hipoplasia del esmalte dental (Fig. 3). Así, tanto el paciente como la madre reunieron criterios mayores suficientes para establecer el diagnóstico de esclerosis tuberosa (Tabla 1).

Durante su estancia presenta crisis recurrentes de cianosis, alcanzando oximetrías por debajo de $50 \%$, por lo cual requirió manejo con oxígeno suplementario por cánula nasal, bolos de cristaloides y medidas de confort. Ante el incremento de la frecuencia y la duración de las crisis se decide realizar cateterismo cardiaco con el fin de evaluar objetivamente los gradientes en el tracto pulmonar y considerar la opción de intervencionismo según los hallazgos. Se corrobora el diagnóstico de tetralogía de Fallot con estenosis infundibulovalvular grave (gradiente $55 \mathrm{mmHg}$ ) y se practica valvuloplastia pulmonar percutánea con resultado exitoso (gradiente $20 \mathrm{mmHg}$ ).

Posterior al procedimiento se observa una franca mejoría en la oximetría, alcanzando saturaciones entre
Tabla 1. Criterios diagnósticos de esclerosis tuberosa

\begin{tabular}{|c|c|}
\hline Criterios mayores & Criterios menores \\
\hline $\begin{array}{l}\text { - Máculas } \\
\text { hipomelanóticas }(\geq 3 \text {, al } \\
\text { menos } 5 \text { mm de } \\
\text { diámetro) } \\
\text { - Angiofibromas }(\geq 3) \text { o } \\
\text { placa cefálica fibrosa } \\
\text { - Fibromas ungueales ( } \geq 2) \\
\text { - Parche de Shagreen } \\
\text { - Hamartomas retinianos } \\
\text { múltiples } \\
\text { - Displasias corticales } \\
\text { - Nódulos } \\
\text { subependimarios } \\
\text { - Astrocitoma } \\
\text { subependimario de } \\
\text { células gigantes } \\
\text { - Rabdomioma cardiaco } \\
\text { - Linfangioleiomiomatosis } \\
\text { - Angiomiolipomas }\end{array}$ & $\begin{array}{l}\text { - Lesiones cutáneas de "confeti» } \\
\text { - Huecos de esmalte dental }(>3) \\
\text { - Fibromas intraorales }(\geq 2) \\
\text { - Parche acrómico retiniano } \\
\text { - Quistes renales múltiples } \\
\text { - Hamartomas no renales }\end{array}$ \\
\hline
\end{tabular}

Diagnóstico definitivo: 2 criterios mayores o 1 criterio mayor +2 criterios menores. Posible diagnóstico: 1 criterio mayor o 2 criterios menores.

* Incluye tubérculos y líneas de migración radial de la materia blanca cerebral.

${ }^{\dagger}$ Cuando estos dos criterios están presentes se requieren otros factores de

esclerosis tuberosa para hacer el diagnóstico definitivo.

Fuente: elaborada por los autores.

el $90 \%$ y el $92 \%$. Se inicia un beta-bloqueador ante el componente de estenosis infundibular descrito, pero el paciente presenta un incremento progresivo de la 


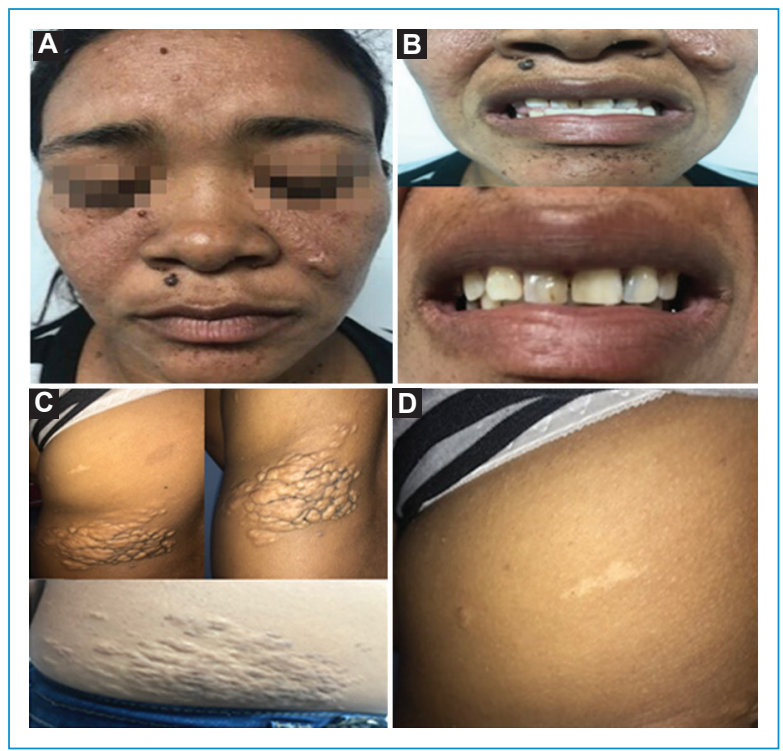

Figura 3. Imágenes de la madre del paciente. Signos de esclerosis tuberosa. A: angiofibromas en el rostro. B: hipoplasia del esmalte dental. C: fibromatosis en la región infraglútea derecha y en la región lumbar baja derecha. D: mácula hipomelánica "en hoja de fresno" en la región glútea derecha

frecuencia cardiaca con salvas de taquicardia de complejos estrechos, inicialmente autolimitada sin repercusión hemodinámica, permaneciendo estable durante 18 horas. Luego, de manera súbita, desarrolla taquicardia supraventricular sin respuesta a la adenosina ni la amiodarona, que lleva a un desenlace fatal.

\section{Discusión}

Los rabdomiomas son los tumores cardiacos primarios más frecuentes en la edad pediátrica, constituyendo más del $60 \%$ de los casos. Están asociados al complejo de esclerosis tuberosa y suelen ser la primera manifestación de esta afección detectable en forma prenatal $^{8,9}$.

La tetralogía de Fallot, el síndrome de corazón izquierdo hipoplásico y la fibroelastosis endocárdica son cardiopatías congénitas que están asociadas con estos tumores sin una relación causa-efecto documenta$\mathrm{da}^{9}$. Las guías internacionales y nacionales recomiendan el tamizaje prenatal de estas alteraciones estructurales cardiacas durante el segundo trimestre a toda embarazada entre las 18 y 24 semanas de gestación por medio de la ecografía obstétrica de detalle anatómi$\mathrm{co}^{10-12}$. Si existieran hallazgos de cardiopatía congénita, está indicado el ecocardiograma fetal, con una precisión diagnóstica de hasta el 96\% (dependiente del operador) ${ }^{13}$. Otra de las indicaciones para su realización es la presencia de factores de riesgo maternos, fetales y familiares, como diabetes mellitus pregestacional, autoanticuerpos maternos, infección por rubeola en el primer trimestre, cardiopatía congénita en pariente de primer grado del feto, translucencia nucal o cariotipo fetal anormal. Los pacientes de alto riesgo pueden requerir una evaluación temprana, generalmente desde la semana 12 de gestación ${ }^{14}$.

En el caso descrito no se incluyó la ecografía de detalle anatómico en el control prenatal, lo cual retardó el diagnóstico y el abordaje en el periodo neonatal; por tanto, se limita la correcta asignación de un centro cardiovascular pediátrico para la atención del parto y del manejo multidisciplinario temprano. Es importante señalar que el diagnóstico prenatal detallado y oportuno permite brindar asesoría completa a los padres, definir el lugar de nacimiento, preparar el equipo multidisciplinario para ofrecer una atención neonatal integral, así como estructurar un plan de tratamiento prenatal y posnatal, de modo que disminuya la tasa de morbimortalidad neonata ${ }^{15}$.

La presentación clínica de los rabdomiomas depende del número, el tamaño y la localización. Son sintomáticos aquellos pacientes cuyas masas generan obstrucción de los tractos de entrada o salida del corazón y arritmias; estas últimas ocurren hasta en un $16-47 \%$ de los casos, describiéndose principalmente síndromes de preexcitación $n^{9,16-18}$. En este paciente, luego de la valvuloplastia pulmonar exitosa y la mejoría de la oximetría posterior al cateterismo cardiaco, se registró arritmia (taquicardia supraventricular) no asociada a síndrome de preexcitación, que no respondió al manejo farmacológico y condujo a un desenlace fatal. Se han descrito la muerte intrauterina y la muerte súbita posnatal, y está indicado el uso temprano de antiarrítmicos cuando la arritmia es el síntoma inicial y la resección de las masas cuando no es posible el control farmacológico ${ }^{19}$.

La ecocardiografía es un excelente método de alta sensibilidad para el diagnóstico de masas cardiacas y la técnica de referencia en el diagnóstico de las cardiopatías congénitas. Permite identificar los rabdomiomas como masas intramurales o intracavitarias relativamente homogéneas, bien circunscritas, con reflejo de eco, ubicadas prácticamente en cualquier estructura cardiaca, aunque con mayor frecuencia en los ventrículos ${ }^{20}$.

La RM cardiaca se reserva para pacientes seleccionados en los que el tipo de tumor es cuestionable 
después de la ecocardiografía o se requiere información anatómica o funcional adicional ${ }^{21}$. La RM también es útil como estudio de extensión, ya que permite evaluar lesiones características de esclerosis tuberosa en otros órganos, como el cerebro, el hígado y los riñones, que pueden verse afectados. En el caso reportado se documentaron nódulos subependimarios en el sistema ventricular lateral, lo que permitió confirmar el diagnóstico de esclerosis tuberosa e iniciar el acompañamiento por parte del servicio de neurología pediátrica ${ }^{19-22}$.

Aunque el manejo expectante es la norma para la mayoría de los tumores, en especial para los rabdomiomas, que tienen una regresión espontánea en el 90\% de los $\operatorname{casos}^{23}$, la resección quirúrgica está indicada para las arritmias refractarias y para el comportamiento hemodinámico «maligno» (mecánico-obstructivo en tractos de entrada o salida que compromete el gasto cardiaco), y es segura y generalmente definitiva ${ }^{9}$, con una mortalidad operatoria descrita del $5 \%$ al $17.5 \%$. De acuerdo con los casos reportados, se han obtenido resultados posquirúrgicos alentadores; aproximadamente el $91 \%$ de los pacientes no requirieron reintervención quirúrgica relacionada con el tumor en un periodo de 10 años, el $85 \%$ presentaron clase funcional I de la New York Heart Association y el $100 \%$ ritmo sinusal ${ }^{9}$.

Numerosos estudios han reportado el uso de everolimus, un medicamento que actúa como inhibidor de la proteína m-TOR, la cual está relacionada con la proliferación y el crecimiento celular excesivo que llevan al desarrollo de hamartomas, tumores benignos asociados a mutaciones que resultan en una deficiencia del complejo tuberina/hamartina, el cual desempeña un papel inhibitorio de la vía $m-T_{O} R^{15,24}$. El everolimus está aprobado por la Food and Drug Administration de los Estados Unidos de América para el tratamiento de astrocitomas de células gigantes en niños. Durante su uso, diversos autores han observado una reducción en el tamaño final de los rabdomiomas con periodos variables de tratamiento que oscilan entre 1 y 4 meses. Chang, et al. ${ }^{25}$ utilizaron dosis menores que la recomendada para el manejo de los astrocitomas en tres neonatos con rabdomiomas grandes, y lograron una reducción del tamaño de los tumores en 2 meses de tratamiento. Los tres pacientes tuvieron complicaciones infecciosas por el efecto inmunosupresor del medicamento, las cuales fueron resueltas favorablemente. Aunque los casos reportados en la literatura sugieren, en general, una relación riesgo/beneficio positiva con el uso del medicamento, se requieren más estudios para evaluar mejor la respuesta terapéutica, así como para determinar los efectos secundarios a largo plazo ${ }^{26-30}$.

\section{Conclusiones}

La asociación de tumores cardiacos con el complejo de esclerosis tuberosa es común; sin embargo, el hallazgo de esta combinación con cardiopatías congénitas es inusual y se describen pocos casos en la literatura, con peor pronóstico y mayor riesgo de mortalidad, como en nuestro caso. Su diagnóstico y manejo constituyen un reto para el equipo tratante. Por fortuna, se cuenta con estudios prenatales y posnatales accesibles, como la ecografía obstétrica de detalle anatómico y el ecocardiograma fetal y posnatal, que ofrecen una sensibilidad diagnóstica alta, lo cual permite estructurar estrategias de manejo individuales de acuerdo con las características clínicas y anatómicas del paciente, impactando así la morbimortalidad neonatal y la sobrevida.

\section{Financiamiento}

Los autores declaran que no recibieron financiamiento para realizar esta publicación.

\section{Conflicto de intereses}

Los autores declaran que no tienen conflicto de intereses para escribir este artículo.

\section{Responsabilidades éticas}

Protección de personas y animales. Los autores declaran que para esta investigación no se han realizado experimentos en seres humanos ni en animales.

Confidencialidad de los datos. Los autores declaran que han seguido los protocolos de su centro de trabajo sobre la publicación de datos de pacientes.

Derecho a la privacidad y consentimiento informado. Los autores han obtenido el consentimiento informado de los pacientes y/o sujetos referidos en el artículo. Este documento obra en poder del autor de correspondencia.

\section{Bibliografía} 1. Tzani A, Doulamis IP, Mylonas KS, Avgerinos DV, Nasioudis D. Cardiac
tumors in pediatric patients: a systematic review. World J Pediatr Con-
genit Hear Surg. $2017 ; 8: 624-32$.
2. Gómez MT, Vargas JA. Esclerosis tuberosa. Revista Médica Sinergia.
2019;4:21-37. 
3. Lince R, Gómez López de Mesa C, Arteaga A, Montoya JH, Vásquez LM. Rabdomioma cardiaco como manifestación de esclerosis tuberosa: presentación de dos casos y revisión de la literatura. Rev Colomb Cardiol. 2009;16:224-8.

4. Jaramillo Daza JF, Cruz Osorio V. Rabdomioma fetal: diagnóstico prenatal y tratamiento. Rev Colomb Cardiol. 2016;23:454.e1-5.

5. Apitz C, Webb GD, Redington AN. Tetralogy of Fallot. Lancet. 2009; 374:1462-71.

6. Muneuchi J, Watanabe M, Sugitani Y, Kawaguchi N, Matsuoka R, Ando Y, et al. Early palliative balloon pulmonary valvuloplasty in neonates and young infants with tetralogy of Fallot. Heart Vessels. 2020;35:252-8.

7. Qin C, Chen L, Wen RG, Xiao YB. Total correction of tetralogy of Fallot associated with rhabdomyoma in the right ventricle. J Thorac Cardiovasc Surg. 2011:141:e20-2.

8. Hinton RB, Prakash A, Romp RL, Krueger DA, Knilans TK. Cardiovascular manifestations of tuberous sclerosis complex and summary of the revised diagnostic criteria and surveillance and management recommendations from the International Tuberous Sclerosis Consensus Group. J Am Heart Assoc. 2014;3:e001493.

9. Yadava OP. Cardiac tumours in infancy. Indian Heart J. 2012;64:492-6.

10. Bravo-Valenzuela N, Borges Peixoto A, Araujo Júnior E, Prenatal diagnosis of congenital heart disease: a review of current knowledge. Indian Heart J. 2018;70:150-64.

11. Donofrio MT, MoonGrady AJ, Hornberger LK, Copel JA, Sklansk MS, Abuhamad A, et al. Diagnosis and treatment of fetal cardiac disease. Circulation. 2014;129:2183-242.

12. Grupo Desarrollador de la Guía - Universidad Nacional de Colombia - Alianza CINETS. Guía de práctica clínica para la prevención, detección temprana y tratamiento del embarazo, parto o puerperio. Rev Colomb Obstet Ginecol. 2015;66:263-86.

13. Allan LD, Chita SK, Sharland GK, Fagg NL, Anderson RH, Crawford DC. The accuracy of fetal echocardiography in the diagnosis of congenital heart disease. Int J Cardiol. 1989;25:279-88.

14. Carvalho JS, Moscoso G, Ville Y. First trimester transabdominal fetal echocardiography. Lancet. 1998;351:1023-7.

15. Krueger DA, Northrup H. International Tuberous Sclerosis Complex Consensus Group. Pediatr Neurol. 2013;49:255-65.

16. Mas C, Penny DJ, Menahem S. Preexcitation syndrome secondary to cardiac rhabdomyomas in tuberous sclerosis. J Paediatric Child Health. $2000 ; 36: 84-6$.
17. Jain D, Maleszewski JJ, Halushka MK. Benign cardiac tumors and tumorlike conditions. Ann Diagn Pathol. 2010;14:215-30.

18. Miyake CY, Del Nido PJ, Alexander ME, Cecchin F, Berul Cl, Triedman JK, et al. Cardiac tumors and associated arrhythmias in pediatric patients, with observations on surgical therapy for ventricular tachycardia. J Am Coll Cardiol. 2011;58:1903-9.

19. Burke A, Virmani R. Pediatric heart tumors. Cardiovasc Pathol. 2008; 17:193-8.

20. Di Liang C, Ko SF, Huang SC. Echocardiographic evaluation of cardiac rhabdomyoma in infants and children. J Clin Ultrasound. 2000;28:381-6.

21. Sugiyama H, Naito H, Tsukano S, Echigo S, Kamiya T. Evaluation of cardiac tumors in children by electron-beam computed tomography: rhabdomyoma and fibroma. Circ J. 2005;69:1352-6.

22. Syed IS, Feng D, Harris SR, Martinez MW, Misselt AJ, Breen JF. MR imaging of cardiac masses. Magn Reson Imaging Clin N Am. 2008;16: 137-64.

23. Freedom RM, Lee KJ, Mac Donald C, Taylor G. Selected aspects of cardiac tumours in infancy and childhood. Pediatr Cardiol. 2000;21: 299-316.

24. Dahdah N. Everolimus for the treatment of tuberous sclerosis complex-related cardiac rhabdomyomas in pediatric patients. J Pediatr. 2017;190:21-26.e7.

25. Chang JS, Chiou PY, Yao SH, Chou IC, Lin CY. Regression of neonatal cardiac rhabdomyoma in two months through low-dose everolimus therapy: a report of three cases. Pediatr Cardiol. 2017;38:1478ロ4.

26. Tiberio D, Franz DN, Phillips JR. Regression of a cardiac rhabdomyoma in a patient receiving everolimus. Pediatrics. 2011;127:e1335-7.

27. Aw F, Goyer I, Raboisson MJ, Boutin C, Major P, Dahdah N. Accelerated cardiac rhabdomyoma regression with everolimus in infants with tuberous sclerosis complex. Pediatr Cardiol. 2017:38:394-400.

28. Hoshal SG, Samuel BP, Schneider JR, Mammen L, Vettukattil JJ. Regression of massive cardiac rhabdomyoma on everolimus therapy. Pediatr Int. 2016:58:397-9.

29. Goyer I, Dahdah N, Major P. Use of mTOR inhibitor everolimus in three neonates for treatment of tumors associated with tuberous sclerosis complex. Pediatr Neurol. 2015;52:450-3.

30. Doğan V, Yeşil Ş, Kayalı Ş, Beken S, Özgür S, Ertuğrul i, et al. Regression of symptomatic multiple cardiac rhabdomyomas associated with tuberous sclerosis complex in a newborn receiving everolimus. J Trop Pediatr. 2015;61:74-7. 